\title{
On the Dirichlet-Neumann Boundary Problem for Scalar Conservation Laws
}

\author{
Marin Mišur ${ }^{a}$, Darko Mitrović ${ }^{b}$ and Andrej Novak ${ }^{c}$ \\ ${ }^{a}$ Faculty of Science, University of Zagreb \\ Bijenička cesta 30, 10000 Zagreb, Croatia \\ ${ }^{b}$ Faculty of Mathematics, University of Montenegro \\ 81000 Podgorica, Montenegro \\ ${ }^{c}$ Faculty of Electrical Engineering, University of Zagreb \\ Unska ulica 3, 10000 Zagreb, Croatia \\ E-mail(corresp.): darkom@ac.me \\ E-mail: mmisur@math.hr, andrej.novak@fer.hr
}

Received July 7, 2015; revised June 26, 2016; published online September 15, 2016

\begin{abstract}
We consider a Dirichlet-Neumann boundary problem in a bounded domain for scalar conservation laws. We construct an approximate solution to the problem via an elliptic approximation for which, under appropriate assumptions, we prove that the corresponding limit satisfies the considered equation in the interior of the domain. The basic tool is the compensated compactness method. We also provide numerical examples.
\end{abstract}

Keywords: scalar conservation law, bounded domain, Dirichlet-Neumann problem, compensated compactness, numerical simulations.

AMS Subject Classification: 35L65; 65N99.

\section{Introduction}

In the paper, we investigate the following mixed boundary problem on an open bounded domain $\Omega \subseteq[0, \infty\rangle \times \mathbf{R}$ with the boundary $\partial \Omega=\Gamma_{N} \dot{\cup} \Gamma_{D}, \Gamma_{D} \subset\{t=$ $0\}$, of class $\mathrm{C}^{0,1}$ :

$$
\begin{aligned}
& \partial_{t} u+\partial_{x}(f(t, x, u))=0 \text { in } \Omega, \\
& \nabla_{(t, x)} u \cdot \nu=0 \text { on } \Gamma_{N}, \\
& u(0, \cdot)=u^{0}(\cdot) \in \mathrm{L}^{\infty}(\mathbf{R}) \text { on } \Gamma_{D},
\end{aligned}
$$

where $\Gamma_{N}$ and $\Gamma_{D} \subset\{t=0\}$ are partitions of $\partial \Omega$ of strictly positive (Hausdorff) measure and $\nu$ is the outer unit normal vector on $\Gamma_{N}$ (see Figure 1). We also assume that $f(t, x, \lambda)$ is a Caratheodory type function i.e. it is of bounded variation with respect to the variables $(t, x)$ and differentiable with respect to the third variable $\lambda$. 


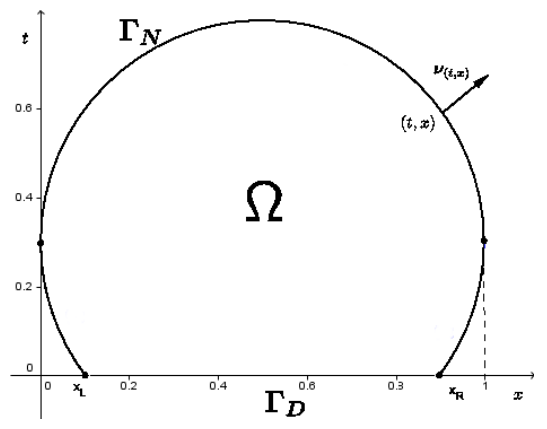

Figure 1. An example of the domain of the problem we investigate.

Here, we are dealing with the pure boundary problem. A similar situation, but under different conditions, was considered in [21] where the Dirichlet boundary problem was considered (even on manifolds). The case of general Neumann conditions $\nabla_{(t, x)} u \cdot \nu=\nabla_{(t, x)} u_{B} \cdot \nu$ on $\Gamma_{N}$ for $u_{B} \in \mathrm{C}\left(\Gamma_{N}\right)$ can be treated by assuming that there exists a continuous function $\tilde{u}_{B} \in \mathrm{C}([0, \infty) \times \mathbf{R})$ such that $\left.\tilde{u}_{B}\right|_{\Gamma_{N}}=u_{B}$. Then, introducing the function $v=u-\tilde{u}_{B}$, we reduce the problem to the one of type (1.2) (though with a non-zero source term).

To proceed, recall that it is more usual to consider initial-boundary value problem for scalar conservation laws. This was first done in [5] and investigations in this directions are quite active (see e.g. $[2,4,11,15,17,26]$ and references therein). Application of such problems are often in traffic flow [25] or filtration and sedimentation models $[7,8]$.

Let us remark that in [7] initial-boundary problem for (1.1) with zero-flux boundary conditions was considered (i.e. no flow through the boundary) and it is sometimes called Neumann problem for (1.1). To explain reasons for the latter, assume that we are considering (1.1) on $\mathbf{R}^{+} \times[a, b]$. After integrating (1.1) over $[a, b]$ and taking into account that $f(t, a, u(t, a))=f(t, b, u(t, b))=0$, we conclude

$$
\frac{d}{d t} \int_{a}^{b} u(t, x) d x=0,
$$

i.e. there is inflow or outflow in the interval $[a, b]$, but the total mass remains the same (it is conserved). Here, we are considering domain which is bounded with respect to both variables $\left(t \in \mathbf{R}^{+}\right.$and $\left.x \in \mathbf{R}\right)$, and we require pointwise control at the boundary. More precisely, we require that the inflow is equal to the outflow in the normal direction of every point $(t, x) \in \partial \Omega$ (unlike the situation from [7] where there was no mass change globally but there can be difference in the inflow and outflow through a boundary point; see also [6,9]). Such problem was not previously considered in the literature.

Motivation for the research was possible application on the traffic flow modelling. Namely, if we consider (1.1) as a model of cars density on the road, the Neumann boundary conditions enable us to have global control on the inflow/outflow of cars with respect to the geometry of the domain. In other 
words, we would like to control density of cars by controlling inflow and outflow with respect both variables, space and time so that the road boundary is permeable with constraint on times-space no-density-change in the direction of normal to the part of the domain (boundary) where Neumann boundary condition is imposed.

Let us now go back to the technical questions. For a function $F=F(t, x, \lambda)$, we will denote by $F_{x}^{\prime}(t, x, \lambda)$ and $F_{\lambda}^{\prime}(t, x, \lambda)$ distributional derivatives with respect to $x$ and $\lambda$ respectively. Similar notations will be used for higher order derivatives. Remark that if $F$ is smooth enough then we have the standard derivative. However, for the flux $f$, the $x$-derivative is actually a measure since $f$ is of bounded variation with respect to $x$. By $\chi_{K}$ we will denote the characteristic function of the set $K$.

Since our main theorem in the next section is of the local nature, it is enough to assume that for every $\xi \in \mathbf{R}$, the functions $\int_{0}^{\xi} f(t, x, \lambda) d \lambda, \int_{0}^{\xi} f_{x}^{\prime}(t, x, \lambda) d \lambda$ and $f(t, x, \lambda)$ belong to some local Lebesgue spaces. More precisely, we assume that function $f$ satisfies the following three assumptions for a fixed $p \in\langle 2, \infty\rangle$ :

- A1: $(\forall \Lambda \subset \mathbf{R}$ compact $)(\forall K \subset \Omega$ compact $)\left(\exists C_{1}=C_{1}(K, \Lambda)>0\right)(\forall \xi \in \Lambda)$

$$
\left\|\chi_{K} \int_{0}^{\xi} f(t, x, \lambda) d \lambda\right\|_{L^{p}(\Omega)}<C_{1},
$$

- A2: $(\forall \Lambda \subset \mathbf{R}$ compact $)(\forall K \subset \Omega$ compact $)\left(\exists C_{2}=C_{2}(K, \Lambda)>0\right)(\forall \xi \in \Lambda)$

$$
\left\|\chi_{K} \int_{0}^{\xi} f_{x}^{\prime}(t, x, \lambda) d \lambda\right\|_{\mathrm{L}^{1}(\Omega)}<C_{2},
$$

where $\mathrm{L}^{1}(\Omega)$ is taken with respect to the measure $\int_{0}^{\xi} f_{x}^{\prime}(t, x, \lambda) d \lambda$,

- A3: $(\forall \Lambda \subset \mathbf{R}$ compact $)(\forall K \subset \Omega$ compact $)\left(\exists C_{3}=C_{3}(K, \Lambda)>0\right)(\forall \lambda \in \Lambda)$

$$
\left\|\chi_{K} f(t, x, \lambda)\right\|_{\mathrm{L}^{p}(\Omega)}<C_{3} .
$$

Notice that assumptions A1 and A3, due to the boundedness of $\Omega$, imply that for every $\Lambda \subset \mathbf{R}$ compact and every $\varphi \in \mathrm{C}_{c}(\Omega)$, the following holds for positive constants $C_{1, p, K, \Lambda}$ and $C_{3, p, K, \Lambda}$ with $K=\operatorname{supp} \varphi$ :

- $\mathrm{C} 1: \quad(\forall \xi \in \Lambda) \quad\left\|\varphi(t, x) \int_{0}^{\xi} f(t, x, \lambda) d \lambda\right\|_{\mathrm{L}^{1}(\Omega)}<C_{1, p, K, \Lambda}\|\varphi\|_{\mathrm{L}^{\infty}(\Omega)}$,

- $\quad \mathrm{C} 3: \quad(\forall \lambda \in \Lambda) \quad\|\varphi(t, x) f(t, x, \lambda)\|_{\mathrm{L}^{1}(\Omega)}<C_{3, p, K, \Lambda}\|\varphi\|_{\mathrm{L}^{\infty}(\Omega)}$.

A natural question that arises is what would be proper solution concept for (1.1), (1.2), (1.3). At this moment, we are not able to introduce appropriate definition which would provide well posedness to $(1.1),(1.2),(1.3)$ in the corresponding sense. However, we shall propose an informal solution concept by considering an elliptic approximation to the problem:

$$
\begin{aligned}
& \partial_{t} u_{n}+\partial_{x}\left(f_{n}\left(t, x, u_{n}\right)\right)=(1 / n) \triangle_{(t, x)} u_{n} \text { in } \Omega, \\
& \nabla_{(t, x)} u_{n} \cdot \nu=0 \text { on } \Gamma_{N}, \\
& u_{n}(0, \cdot)=u_{n}^{0}(\cdot) \text { on } \Gamma_{D},
\end{aligned}
$$


where $f_{n}(t, x, \lambda)=f(\cdot, \cdot, \lambda) \star n^{2} \omega(n t, n x)$ is a regularization of the flux $f$ via the standard non-negative mollifier $\omega \in \mathrm{C}_{c}^{\infty}\left((-1,1)^{2}\right)$ with total mass one, and $\left(u_{n}^{0}\right)$ is a bounded sequence of functions converging strongly in $\mathrm{L}_{l o c}^{1}(\mathbf{R})$ toward $u_{0}$. By multiplying (1.4) by $\operatorname{sgn}\left(u_{n}(t, x)-\lambda\right)$ we get after standard manipulations (see also formula (2.8) of the next section):

$$
\begin{aligned}
\partial_{t}\left|u_{n}-\lambda\right|+\partial_{x} & \left(\operatorname{sgn}\left(u_{n}-\lambda\right)\left(f_{n}\left(t, x, u_{n}\right)-f_{n}(t, x, \lambda)\right)\right. \\
\leq & \frac{1}{n} \triangle_{(t, x)}\left|u_{n}-\lambda\right|-\operatorname{sgn}\left(u_{n}-\lambda\right) f_{n, x}^{\prime}(t, x, \lambda) \text { in } \Omega .
\end{aligned}
$$

We multiply the latter equation by $\varphi \in \mathrm{C}^{1}(\Omega)$ supported away from $\{t=0\}$ and integrate over $\Omega$. After taking into account (1.2), we get:

$$
\begin{aligned}
& -\int_{\Omega}\left(\left|u_{n}-\lambda\right| \partial_{t} \varphi+\operatorname{sgn}\left(u_{n}-\lambda\right)\left(f_{n}\left(t, x, u_{n}\right)-f_{n}(t, x, \lambda)\right) \partial_{x} \varphi\right) d x d t \\
& \quad+\int_{\partial \Omega}\left(\left|u_{n}-\lambda\right|, \operatorname{sgn}\left(u_{n}-\lambda\right)\left(f_{n}\left(t, x, u_{n}\right)-f_{n}(t, x, \lambda)\right)\right) \cdot \nu \varphi d s \\
& \quad \leq \frac{1}{n} \int_{\Omega} \nabla_{(t, x)}\left|u_{n}-\lambda\right| \cdot \nabla_{(t, x)} \varphi d x d t-\int_{\Omega} \varphi \operatorname{sgn}\left(u_{n}-\lambda\right) f_{n, x}^{\prime}(t, x, \lambda) d x d t .
\end{aligned}
$$

Now, we assume that $\frac{1}{n} \nabla_{(t, x)}\left|u_{n}-\lambda\right| \rightarrow 0$ in $\mathrm{L}^{2}(\Omega)$ which is the case when dealing with the Cauchy problem for the vanishing viscosity approximation of scalar conservation laws [13, pages 58,59] (also see the proof in the next section). The second term on the right hand side is bounded due to assumption A2. Thus, the right-hand side of (1.5) is uniformly bounded. If we assume that $u_{n} \rightarrow u$ strongly in $\mathrm{L}^{1}(\Omega)$, we know what will happen with the first term on the left-hand side of (1.5).

The remaining second term on the left hand side of (1.5) depends on the behaviour of $u_{n}$ on the boundary of $\Omega$. We do not have that information and we will use the idea from Formula (7), page 1310. in [3] (where a similar problem of controlling the viscosity approximation on a zero measure set appear and where it is successfully resolved) to introduce the following definition.

Definition 1. The function $u \in \mathrm{L}^{2}(\Omega)$ is called a solution to (1.1), (1.2), (1.3) if there exists a function $p \in \mathrm{L}^{1}\left(\Gamma_{N}\right)$ such that for every $\varphi \in \mathrm{C}_{c}^{1}\left(\bar{\Omega} \backslash \Gamma_{D}\right)$ that the following holds:

$$
\begin{aligned}
\int_{\Omega}(\mid & \left.-\lambda \mid \partial_{t} \varphi+\operatorname{sgn}(u-\lambda)(f(t, x, u)-f(t, x, \lambda)) \partial_{x} \varphi\right) d x d t \\
& -\int_{\partial \Omega}(|p-\lambda|, \operatorname{sgn}(p-\lambda)(f(t, x, p)-f(t, x, \lambda))) \cdot \nu \varphi d s \\
& \geq \int_{\Omega} \varphi \operatorname{sgn}(u-\lambda) f_{x}^{\prime}(t, x, \lambda) d x d t .
\end{aligned}
$$

Initial data are satisfied in the strong sense i.e. for almost every $x \in \Gamma_{D}$ it holds $\lim _{t \rightarrow 0}\left|u(t, x)-u_{0}(x)\right|=0$.

We are not able to prove existence or uniqueness of function satisfying conditions of Definition 1. Instead, in the next section, we shall prove that 
under appropriate assumptions, the sequence $\left(u_{n}\right)$ defined by $(1.4)$ weakly converges toward $u$ satisfying (1.6) in the interior of $\Omega$ i.e. for the test functions $\varphi \in \mathrm{C}_{c}(\Omega)$. The main tool that we are going to use for this purpose is the celebrated compensated compactness method [12,19,27]. In Section 3, we shall examine numerical convergence of the approximation (1.4) in a special situation and provide appropriate simulations.

\section{The main result}

As in the case of the diffusion-dispersion limit for scalar conservation laws (see e.g. $[1,16,24]$ and references therein), it is little known about existence of solution to (1.4). Therefore, we need to assume certain properties of the sequence of solutions $\left(u_{n}\right)$ to $(1.4)$. In the final section, we shall provide simulations which show that the sequence of approximate solutions stays uniformly bounded (in the special case of the Burgers equation). Under such assumptions, we are able to prove that the sequence $\left(u_{n}\right)$ converges toward the distributional solution $u$ to (1.1) in the interior of $\Omega$. Moreover, according to the results from $[20$, Remark 1], the solution $u$ satisfies also the Kruzhkov entropy conditions in the interior of $\Omega$. The following theorem holds:

Theorem 1. Assume that the sequence $\left(u_{n}\right)$ of solutions to (1.4) is uniformly bounded by a constant $M$. If the flux $f$ satisfies the assumptions A1, A2 and $A 3$, then the weak $\mathrm{L}^{2}(\Omega)$-limit of $\left(u_{n}\right)$ along a subsequence satisfies the equation (1.1) in $\Omega$.

Proof. Let us denote by $u$ a weak $\mathrm{L}^{2}(\Omega)$-limit of $\left(u_{n}\right)$ along some subsequence. Take a convex entropy $\Phi: \mathbf{R} \rightarrow \mathbf{R}$ such that $\Phi^{\prime}(0)=0$, and multiply the first equation in (1.4) with $\Phi^{\prime}\left(u_{n}\right)$. Before we proceed, let us remark that we can write

$$
\partial_{x}\left(f_{n}\left(t, x, u_{n}\right)\right)=f_{n, x}^{\prime}\left(t, x, u_{n}\right)+f_{n, \lambda}^{\prime}\left(t, x, u_{n}\right) \partial_{x} u_{n},
$$

so the equation in (1.4) becomes:

$$
\begin{gathered}
\partial_{t} u_{n} \Phi^{\prime}\left(u_{n}\right)+f_{n, x}^{\prime}\left(t, x, u_{n}\right) \Phi^{\prime}\left(u_{n}\right)+f_{n, \lambda}^{\prime}\left(t, x, u_{n}\right) \partial_{x} u_{n} \Phi^{\prime}\left(u_{n}\right) \\
=\partial_{t}\left(\Phi\left(u_{n}\right)\right)+f_{n, x}^{\prime}\left(t, x, u_{n}\right) \Phi^{\prime}\left(u_{n}\right)+\partial_{x}\left(\int_{0}^{u_{n}} f_{n, \lambda}^{\prime}(t, x, \lambda) \Phi^{\prime}(\lambda) d \lambda\right) \\
-\int_{0}^{u_{n}} f_{n, \lambda x}^{\prime \prime}(t, x, \lambda) \Phi^{\prime}(\lambda) d \lambda=\frac{1}{n} \triangle_{(t, x)} u_{n} \Phi^{\prime}\left(u_{n}\right) .
\end{gathered}
$$

An integration by parts, together with the fact that $\Phi^{\prime}(0)=0$, yields the following:

$$
f_{n, x}^{\prime}\left(t, x, u_{n}\right) \Phi^{\prime}\left(u_{n}\right)-\int_{0}^{u_{n}} f_{n, \lambda x}^{\prime \prime}(t, x, \lambda) \Phi^{\prime}(\lambda) d \lambda=\int_{0}^{u_{n}} f_{n, x}^{\prime}(t, x, \lambda) \Phi^{\prime \prime}(\lambda) d \lambda,
$$

while the term on the right hand side in (2.1), we can break into two terms using the following formula:

$$
\triangle_{(t, x)}\left(\Phi\left(u_{n}\right)\right)=\Phi^{\prime \prime}\left(u_{n}\right)\left(\left(\partial_{t} u_{n}\right)^{2}+\left(\partial_{x} u_{n}\right)^{2}\right)+\Phi^{\prime}\left(u_{n}\right) \triangle_{(t, x)} u_{n} .
$$


Using formulae (2.2) and (2.3), the equation (2.1) gets the following form:

$$
\begin{array}{r}
\partial_{t}\left(\Phi\left(u_{n}\right)\right)+\partial_{x}\left(\Psi_{n}\left(t, x, u_{n}\right)\right)+\int_{0}^{u_{n}} f_{n, x}^{\prime}(t, x, \lambda) \Phi^{\prime \prime}(\lambda) d \lambda \\
=\frac{1}{n} \triangle_{(t, x)}\left(\Phi\left(u_{n}\right)\right)-\frac{1}{n} \Phi^{\prime \prime}\left(u_{n}\right)\left|\nabla_{(t, x)} u_{n}\right|^{2}
\end{array}
$$

where the entropy flux $\Psi$ is given by

$$
\Psi_{n}\left(t, x, u_{n}\right)=\int_{0}^{u_{n}} f_{n, \lambda}^{\prime}(t, x, \lambda) \Phi^{\prime}(\lambda) d \lambda,
$$

which, after integration by parts, becomes:

$$
\Psi_{n}\left(t, x, u_{n}\right)=f_{n}\left(t, x, u_{n}\right) \Phi^{\prime}\left(u_{n}\right)-\int_{0}^{u_{n}} f_{n}(t, x, \lambda) \Phi^{\prime \prime}(\lambda) d \lambda .
$$

Now, take $\Phi(\lambda)=\frac{\lambda^{2}}{2}$. Rearranging the terms in the equation (2.4), multiplying it with a nonnegative test function $\varphi(t, x) \in \mathrm{C}_{c}^{2}(\Omega)$ and integrating it over $\Omega$, we get the following bound

$$
\begin{aligned}
& \frac{1}{n} \int_{\Omega}\left|\nabla_{(t, x)} u_{n}\right|^{2} \varphi(t, x) d t d x=\int_{\Omega}\left[\frac{1}{2 n} u_{n}^{2} \triangle_{(t, x)} \varphi+\frac{1}{2} u_{n}^{2} \partial_{t} \varphi\right. \\
& \left.+\Psi_{n}\left(t, x, u_{n}\right) \partial_{x} \varphi-\varphi \int_{0}^{u_{n}} f_{n, x}^{\prime}(t, x, \lambda) d \lambda\right] d t d x<C_{\varphi}<\infty
\end{aligned}
$$

where the first and the second term on the right hand side are bounded due to the assumption of the theorem on uniform boundedness of the sequence $\left(u_{n}\right)$. Furthermore, the last term on the right hand side is bounded due to assumption $\mathrm{A} 2$ and the third term of the right hand side can be rewritten in the following way

$$
\int_{\Omega} \partial_{x} \varphi \Psi_{n}\left(t, x, u_{n}\right) d t d x=\int_{\Omega} \partial_{x} \varphi\left[f_{n}\left(t, x, u_{n}\right) u_{n}-\int_{0}^{u_{n}} f_{n}(t, x, \lambda) d \lambda\right] d t d x .
$$

From here, we see that the term $\int_{\Omega} \partial_{x} \varphi \Psi_{n}\left(t, x, u_{n}\right) d t d x$ is bounded due to the assumption on the uniform bound of the sequence $\left(u_{n}\right)$ and assumptions $\mathrm{C} 1$ and $\mathrm{C} 3$.

Thus, from (2.6), we conclude that $\frac{1}{n} \int_{\Omega}\left|\nabla_{(t, x)} u_{n}\right|^{2} \varphi(t, x) d t d x$ is uniformly bounded for every $\varphi \in \mathrm{C}_{c}^{2}(\Omega)$ and this bound depends on $\varphi$, which implies that $\left(\frac{1}{\sqrt{n}} \nabla_{(t, x)} u_{n}\right)_{n}$ has a weakly convergent subsequence (not relabelled) in $\mathrm{L}_{l o c}^{2}(\Omega)$. Dividing with $\sqrt{n}$, we conclude that $\frac{1}{n} \nabla_{(t, x)} u_{n} \longrightarrow 0$ in $\mathrm{L}_{l o c}^{2}(\Omega)$ and since $\operatorname{div}_{(t, x)}: \mathrm{L}_{l o c}^{2}(\Omega) \rightarrow \mathrm{H}_{l o c}^{-1}(\Omega)$ is continuous, we have $\frac{1}{n} \triangle_{(t, x)} u_{n} \longrightarrow 0$ in $\mathrm{H}_{l o c}^{-1}(\Omega)$. From (1.4), we read

$$
\partial_{t} u_{n}+\partial_{x}\left(f_{n}\left(t, x, u_{n}\right)\right) \longrightarrow 0 \quad \text { in } \mathrm{H}_{l o c}^{-1}(\Omega)
$$

and since $\sup _{\lambda \in(-M, M)}\left|f_{n}(t, x, \lambda)-f(t, x, \lambda)\right| \rightarrow 0$ as $n \rightarrow \infty$ in $\mathrm{L}_{l o c}^{1}(\Omega)$, we conclude

$$
\partial_{t} u_{n}+\partial_{x}\left(f\left(t, x, u_{n}\right)\right) \longrightarrow 0 \text { in } \mathrm{H}_{l o c}^{-1}(\Omega) .
$$


Let us go back at the beginning to the equation (2.4) and assume that $\Phi^{\prime}$ is bounded; rearranging the terms we get:

$$
\begin{aligned}
\partial_{t}\left(\Phi\left(u_{n}\right)\right)+\partial_{x}\left(\Psi_{n}\left(t, x, u_{n}\right)\right)=\frac{1}{n} \triangle_{(t, x)} & \left(\Phi\left(u_{n}\right)\right)-\frac{1}{n} \Phi^{\prime \prime}\left(u_{n}\right)\left|\nabla_{(t, x)} u_{n}\right|^{2} \\
& -\int_{0}^{u_{n}} f_{n, x}^{\prime}(t, x, \lambda) \Phi^{\prime \prime}(\lambda) d \lambda
\end{aligned}
$$

Note that the first term on the right hand side is in $\mathrm{H}_{l o c}^{-1}(\Omega)$. Indeed, taking a test function $\varphi \in \mathrm{H}_{c}^{1}(\Omega)$, we have

$$
\begin{aligned}
\mid \frac{1}{n} \int_{\Omega} & \triangle_{(t, x)}\left(\Phi\left(u_{n}\right)\right) \varphi d t d x|=| \frac{1}{n} \int_{\Omega} \nabla_{(t, x)}\left(\Phi\left(u_{n}\right)\right) \cdot \nabla_{(t, x)} \varphi d t d x \mid \\
& \leq \frac{1}{n} \int_{\Omega}\left|\nabla_{(t, x)}\left(\Phi\left(u_{n}\right)\right)\right|\left|\nabla_{(t, x)} \varphi\right| d t d x \\
& \leq \frac{1}{\sqrt{n}} \int_{\Omega}\left|\frac{1}{\sqrt{n}} \Phi^{\prime}\left(u_{n}\right) \nabla_{(t, x)} u_{n}\right|\left|\nabla_{(t, x)} \varphi\right| d t d x \\
& \leq \frac{1}{\sqrt{n}}\left\|\frac{\chi_{\operatorname{supp} \varphi}}{\sqrt{n}} \Phi^{\prime}\left(u_{n}\right) \nabla_{(t, x)} u_{n}\right\|_{\mathrm{L}^{2}(\Omega)}\left\|\nabla_{(t, x)} \varphi\right\|_{\mathrm{L}^{2}(\Omega)} \leq \frac{C}{\sqrt{n}}\|\varphi\|_{\mathrm{H}^{1}(\Omega)},
\end{aligned}
$$

where we have used Cauchy-Schwartz inequality in the forth step, and boundedness of $\Phi^{\prime}$ and $L^{2}(\Omega)$ bound of $\frac{1}{\sqrt{n}} \nabla_{(t, x)} u_{n} \chi_{\operatorname{supp} \varphi}$ in the last step. Passing to the limit, we see that $\left(\frac{1}{n} \triangle_{(t, x)}\left(\Phi\left(u_{n}\right)\right)\right)_{n}$ is precompact in $\mathrm{H}_{l o c}^{-1}(\Omega)$ and its weak limit is 0 .

Regarding the second term of the right-hand side in (2.8), we have

$$
\left\|\frac{1}{n} \Phi^{\prime \prime}\left(u_{n}\right)\left|\nabla_{(t, x)} u_{n}\right|^{2} \varphi^{2}\right\|_{L^{1}(\Omega)} \leq \tilde{c}\left\|\frac{1}{\sqrt{n}}\left|\nabla_{(t, x)} u_{n}\right| \varphi\right\|_{L^{2}(\Omega)}^{2} \leq \tilde{C}_{\varphi}
$$

for every nonnegative test function $\varphi \in \mathrm{C}_{\mathrm{c}}(\Omega)$, where we have used the $\mathrm{L}^{2}(\Omega)$ bound of $\frac{1}{\sqrt{n}} \nabla_{(t, x)} u_{n} \varphi$ in the last step and the fact that second derivative of a convex function is a nonnegative measure. Since $\mathrm{L}^{1}(\Omega)$ is continously embedded in $\mathcal{M}_{b}(\Omega)$, the space of bounded Radon measures, which in turn is compactly embedded in $\mathrm{W}^{-1, q}(\Omega)$ for all $q \in\langle 1,2\rangle$ [ [13], Theorem 1.6], we conclude that $\left(\frac{1}{n} \Phi^{\prime \prime}\left(u_{n}\right)\left|\nabla_{(t, x)} u_{n}\right|^{2} \varphi^{2}\right)_{n}$ is precompact in $\mathrm{W}^{-1, q}(\Omega), q \in\langle 1,2\rangle$, for every $\varphi \in$ $\mathrm{C}_{c}(\Omega)$. Thus, $\left(\frac{1}{n} \Phi^{\prime \prime}\left(u_{n}\right)\left|\nabla_{(t, x)} u_{n}\right|^{2}\right)_{n}$ is precompact in $\mathrm{W}_{l o c}^{-1, q}(\Omega), q \in\langle 1,2\rangle$.

Now, we turn our attention to the third term of the right hand side of (2.8). Using the nonnegativity of $\Phi^{\prime \prime}$ and assumption A2, we can conclude, in a similar manner as in the case of the second term above, that $\left(\int_{0}^{u_{n}} f_{n, x}^{\prime}(t, x, \lambda) \Phi^{\prime \prime}(\lambda) d \lambda\right)_{n}$ is precompact in $\mathrm{W}_{l o c}^{-1, q}(\Omega), q \in\langle 1,2\rangle$.

In order to show that the sum of the last two terms on the right hand side is precompact in $\mathrm{H}_{l o c}^{-1}(\Omega)$, we will show that their sum is bounded in $\mathrm{W}_{l o c}^{-1, p}(\Omega)$, for $p>2$ from assumptions A1 and A3, and use generalized version of Murat's lemma [ [19], Theorem 2.3.2]. To this end, take a nonnegative test-function 
$\varphi \in \mathrm{C}_{c}^{1}(\Omega)$, and get:

$$
\begin{aligned}
& \left|\int_{\Omega} \partial_{t}\left(\Phi\left(u_{n}\right)\right) \varphi(t, x) d t d x\right|=\left|\int_{\Omega} \Phi\left(u_{n}\right) \partial_{t} \varphi(t, x) d t d x\right| \\
& \quad \leq\left\|\Phi\left(u_{n}\right) \chi_{\operatorname{supp} \varphi}\right\|_{\mathrm{L}^{p}(\Omega)}\left\|\partial_{t} \varphi\right\|_{\mathrm{L}^{p^{\prime}}(\Omega)} \\
& \quad \leq|\Omega| \max _{\lambda \in[-M, M]}|\Phi(\lambda)|\|\varphi\|_{\mathrm{W}^{1, p^{\prime}}(\Omega)} \leq C\|\varphi\|_{\mathrm{W}^{1, p^{\prime}}(\Omega)},
\end{aligned}
$$

where we have used integration by parts in the first step and Hölder inequality in the second one; in the third step, we have used the fact that a convex function defined over whole $\mathbf{R}$ must be continuous and the assumption on the uniform boundedness of the sequence $\left(u_{n}\right)$. Similarly, we have

$$
\begin{aligned}
& \left|\int_{\Omega} \partial_{x}\left(\Psi_{n}\left(t, x, u_{n}\right)\right) \varphi(t, x) d t d x\right|=\left|\int_{\Omega} \Psi_{n}\left(t, x, u_{n}\right) \partial_{x} \varphi(t, x) d t d x\right| \\
& \leq\left|\int_{\Omega} f_{n}\left(t, x, u_{n}\right) \Phi^{\prime}\left(u_{n}\right) \partial_{x} \varphi d t d x\right|+\left|\int_{\Omega} \partial_{x} \varphi \int_{0}^{u_{n}} f_{n}(t, x, \lambda) \Phi^{\prime \prime}(\lambda) d \lambda d t d x\right| \\
& \leq\left\|f_{n}\left(t, x, u_{n}\right) \Phi^{\prime}\left(u_{n}\right) \chi_{\operatorname{supp} \varphi}\right\|_{\mathrm{L}^{p}(\Omega)}\left\|\partial_{x} \varphi\right\|_{\mathrm{L}^{p^{\prime}}(\Omega)}+\left|\int_{\Omega} \partial_{x} \varphi \int_{0}^{u_{n}} f_{n}(t, x, \lambda) d \lambda d t d x\right| \\
& \leq C_{\varphi}\|\varphi\|_{\mathrm{W}^{1, p^{\prime}}(\Omega)}+\left\|\partial_{x} \varphi\right\|_{\mathrm{L}^{p^{\prime}}(\Omega)}\left\|\chi_{\operatorname{supp} \varphi} \int_{0}^{u_{n}} f_{n}(t, x, \lambda) d \lambda\right\|_{\mathrm{L}^{p}(\Omega)} \\
& \leq \tilde{C}_{\varphi}\|\varphi\|_{\mathrm{W}^{1, p^{\prime}}(\Omega)} \cdot
\end{aligned}
$$

In the first step we have used integration by parts and in the second one the expression for the flux from (2.5); in the third step we have used Höler inequality and the non-negativity of $\Phi^{\prime \prime}$, while in the fourth we have used boundedness of $\Phi^{\prime}$ and assumption A3 for the first integral, and Hölder inequality for the second integral; in the last step we have used assumption A1. Let us remark that this is the only place in the proof where we had used assumptions A1 and A3 since we could not have used $\mathrm{C} 1$ and $\mathrm{C} 3$ for the $\mathrm{L}^{p}(\Omega)$ bounds of the above expressions we had.

So far we have shown that the sequence $\left(\partial_{t}\left(\Phi\left(u_{n}\right)\right)+\partial_{x}\left(\Psi_{n}\left(t, x, u_{n}\right)\right)\right)_{n}$ is precompact in $\mathrm{W}_{l o c}^{-1, q}(\Omega), q \in\langle 1,2\rangle$, and bounded in $\mathrm{W}_{l o c}^{-1, p}(\Omega)$ for a $p>2$. The generalised version of Murat's lemma now implies that

$$
\left(\partial_{t}\left(\Phi\left(u_{n}\right)\right)+\partial_{x}\left(\Psi_{n}\left(t, x, u_{n}\right)\right)\right)_{n} \text { is precompact in } \mathrm{H}_{l o c}^{-1}(\Omega),
$$

for all entropy-entropy flux pairs $\left(\Phi(\lambda), \Psi_{n}(t, x, \lambda)\right)$. Specifically, this is valid for the particular entropy-entropy flux pairs which we will use in the rest of the proof - the Kruzhkov entropy-entropy flux pairs. For $k \in \mathbf{R}$, define

$$
\Phi(\lambda)=|\lambda-k|, \quad \Psi_{n}(t, x, \lambda)=\operatorname{sgn}(\lambda-k)\left(f_{n}(t, x, \lambda)-f_{n}(t, x, k)\right) .
$$

As in the case of (2.7), we know that for such defined $\Psi_{n}$ it holds

$$
\sup _{\lambda \in(-M, M)}\left|\Psi_{n}(t, x, \lambda)-\Psi(t, x, \lambda)\right| \rightarrow 0 \text { as } n \rightarrow \infty
$$


in $\mathrm{L}_{l o c}^{1}(\Omega)$, where

$$
\Psi(t, x, \lambda)=\operatorname{sgn}(\lambda-k)(f(t, x, \lambda)-f(t, x, k)) .
$$

Therefore from (2.9) it follows

$$
\begin{array}{r}
\left(\partial_{t}\left|u_{n}-k\right|+\partial_{x}\left(\operatorname{sgn}\left(u_{n}-k\right)\left(f\left(t, x, u_{n}\right)-f(t, x, k)\right)\right)\right)_{n} \\
\text { is precompact in } \mathrm{H}_{l o c}^{-1}(\Omega) .
\end{array}
$$

In the final step we will use the theory of Young measures and the divrot lemma [ [13], Theorem 5.4]. For a.e. $(t, x) \in \Omega$ denote by $\eta_{t, x}$ the Young measure on $\mathbf{R}$ associated to the weak convergence $u_{n} \rightarrow u$ in $\mathrm{L}^{2}(\Omega)$. Since $f$ is the Caratheodory type function we have the following representation (for a.e. $(t, x) \in \Omega)$ :

$$
u(t, x)=\int_{\mathbf{R}} \lambda d \eta_{t, x}(\lambda)
$$

and the following weak convergences in $\mathrm{L}^{2}(\Omega)$ (note that $\Omega$ is bounded):

$$
f\left(\cdot, u_{n}\right) \rightarrow \bar{f}, \quad \Phi\left(u_{n}\right) \rightarrow \bar{\Phi} \quad \text { and } \quad \Psi\left(\cdot, u_{n}\right) \rightarrow \bar{\Psi}
$$

where

$$
\begin{gathered}
\bar{f}(t, x)=\int_{\mathbf{R}} f(t, x, \lambda) d \eta_{t, x}(\lambda), \quad \bar{\Phi}(t, x)=\int_{\mathbf{R}} \Phi(\lambda) d \eta_{t, x}(\lambda) \\
\text { and } \bar{\Psi}(t, x)=\int_{\mathbf{R}} \Psi(t, x, \lambda) d \eta_{t, x}(\lambda) .
\end{gathered}
$$

Remembering the (2.7) and (2.10) and applying the div-rot lemma (div and rot with respect to variables $(t, x))$ on the vector fields $v_{k}=\left(u_{n}, f\left(\cdot, u_{n}\right)\right)$ and $w_{k}=\left(\Psi\left(\cdot, u_{n}\right),-\Phi\left(u_{n}\right)\right)$, we get that $v_{k} \cdot w_{k} \rightarrow v \cdot w$ in the sense of distributions on $\Omega$, where $v=(u, \bar{f})$ and $w=(\bar{\Psi},-\bar{\Phi})$. Using the above representation of $u$ and the weak limits, we conclude the following identity (for a.e. $(t, x) \in \Omega$ )

$$
\begin{aligned}
u(t, x) \bar{\Psi}(t, x)-\bar{f}(t, x) \bar{\Phi}(t, x) & =u(t, x) \int_{\mathbf{R}} \Psi(t, x, \lambda) d \eta_{t, x}(\lambda) \\
-\bar{f}(t, x) \int_{\mathbf{R}} \Phi(\lambda) d \eta_{t, x}(\lambda) & =\int_{\mathbf{R}}(\lambda \Psi(t, x, \lambda)-f(t, x, \lambda) \Phi(\lambda)) d \eta_{t, x}(\lambda) .
\end{aligned}
$$

Rearranging the terms, we can write

$$
\int_{\mathbf{R}}[(f(t, x, \lambda)-\bar{f}(t, x)) \Phi(\lambda)+(u(t, x)-\lambda) \Psi(t, x, \lambda)] d \eta_{t, x}(\lambda)=0 .
$$

Plugging in the particular form of $\Phi$ and $\Psi$, we get the following

$$
\begin{aligned}
0=\int_{\mathbf{R}}[ & (f(t, x, \lambda)-\bar{f}(t, x))|\lambda-k| \\
& +(u(t, x)-\lambda) \operatorname{sgn}(\lambda-k)(f(t, x, \lambda)-f(t, x, k))] d \eta_{t, x}(\lambda) .
\end{aligned}
$$


Taking $k=u(t, x)$, we finally arrive at (for a.e. $(t, x) \in \Omega$ ):

$$
\begin{aligned}
0 & =\int_{\mathbf{R}}[f(t, x, u(t, x))-\bar{f}(t, x)]|\lambda-u(t, x)| d \eta_{t, x}(\lambda) \\
& =[f(t, x, u(t, x))-\bar{f}(t, x)] \int_{\mathbf{R}}|\lambda-u(t, x)| d \eta_{t, x}(\lambda),
\end{aligned}
$$

from which we conclude that either $f(t, x, u(t, x))=\bar{f}(t, x)$ or $\eta_{t, x}=\delta_{u(t, x)}$ for a.e. $(t, x) \in \Omega$. Remembering the definition of $\bar{f}(t, x)$, we see that the second possibility implies the first one. Thus, $f(t, x, u(t, x))=\bar{f}(t, x)$ for a.e. $(t, x) \in \Omega$. In other words, $f(\cdot, u)$ is a weak limit of $f\left(\cdot, u_{n}\right)$. From here, we conclude that $u$ is the weak solution of (1.1) in $\Omega$. This finishes the proof.

A corollary of the proof of the last theorem and [20, Remark 1] in the case when the flux is continuously differentiable with respect to all variables is the fact that the limiting function $u$ satisfies the Kruzhkov admissibility conditions in the interior of $\Omega$ i.e. condition (1.6) from Definition 1 for $\varphi \in \mathrm{C}_{c}^{1}(\Omega)$.

Corollary 1. Assume that the flux $f \in C^{1}(\Omega \times(-M, M))$. The distributional limit $u$ of the sequence $\left(u_{n}\right)$ of solutions to (1.4) satisfies for every entropyentropy flux pair $(\Phi, \Psi)$

$$
\partial_{t}(\Phi(u))+\partial_{x}(\Psi(t, x, u)) \leq-\int_{0}^{u} f_{x}^{\prime}(t, x, \lambda) \Phi^{\prime \prime}(\lambda) d \lambda \text { in } \mathcal{D}^{\prime}(\Omega) .
$$

Proof. The statement follows from (2.8) and [20, Remark 1].

\section{Numerical experiment}

In this section we want to present motivation emanating from the traffic flow modelling, results of the numerical experiment and to briefly outline the technical issues regarding the implementation of numerical method that has been used. First, let us recall Lighthill-Whitham-Richards model for traffic flow $[18,23]$

$$
\partial_{t} \rho+\partial_{x}(\rho v(\rho))=0
$$

in which the velocity is assumed to have linear dependence upon density of the cars

$$
v(\rho)=v_{\max }\left(1-\rho / \rho_{\max }\right), \quad 0 \leq \rho \leq \rho_{\max } .
$$

Let $L$ and $\tau$ be a typical length and time, respectively, such that $v_{\max }=L / \tau$. Introducing new variables $\bar{x}=x / L, \bar{t}=x / L, u=1-2 \rho / \rho_{\max }$, we obtain the inviscid Burgers equation

$$
\partial_{t} \rho+\partial_{x}\left[\rho\left(1-\frac{\rho}{\rho_{\max }}\right)\right]=-\frac{\rho_{\max }}{2 \tau} \partial_{\bar{t}} u-\frac{\rho_{\max }}{2 \tau} \partial_{\bar{x}}\left(\frac{u^{2}}{2}\right)=0 .
$$

For other models, see e.g. $[10,14,22]$ and references therein. 
Let $\Omega=\left\{(t, x) \in \mathbf{R}^{2}: 0 \leq x \leq 1,0 \leq t \leq-4 x(x-1)\right\}$. We focus on solving the (regularized) Burgers equation

$$
\begin{aligned}
& \partial_{t} u+\partial_{x}\left(u^{2} / 2\right)=\epsilon \Delta_{(t, x)} u \text { in } \Omega, \\
& \nabla_{(t, x)} u \cdot \nu=0 \quad \text { on } \Gamma_{N}, \\
& u(0, x)=u_{D} \quad \text { on } \Gamma_{D},
\end{aligned}
$$

where $\Gamma_{D}=\{(t, x) \in \partial \Omega: t=0\}$ and $\Gamma_{N}=\partial \Omega \backslash \Gamma_{D}$ and $u_{D}$ will be specified latter, because it will differ in each out of three experiments. Since we did not make any distinction between space and time, we will use finite element method in space and time. This, rather unusual approach becomes natural if we switch perspective and think of time variable as another space variable.

Let $V_{D}(\Omega)=\left\{v \in \mathrm{H}^{1}(\Omega):\left.v\right|_{\Gamma_{D}}=u_{D}\right\}$ and $\mathrm{H}_{D}^{1}(\Omega)=\left\{v \in \mathrm{H}^{1}(\Omega)\right.$ : $\left.\left.v\right|_{\Gamma_{D}}=0\right\}$. We use the following numerical scheme. For given initial guess $u_{0}$, construct sequence $u_{n} \in V_{D}, n \geq 1$, that are solutions of

$$
\int_{\Omega}\left(\partial_{t} u_{n}+u_{n-1} \partial_{x} u_{n}\right) \psi d t d x+\epsilon \int_{\Omega} \nabla_{(t, x)} u_{n} \cdot \nabla_{(t, x)} \psi d t d x=0, \quad \forall \psi \in \mathrm{H}_{D}^{1}(\Omega)
$$

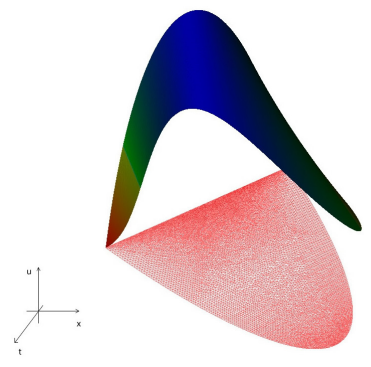

a)

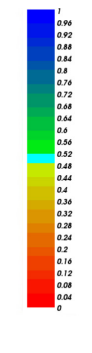

)

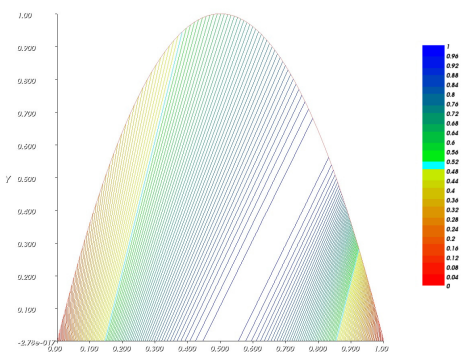

b)

Figure 2. Numerical solution of elliptic approximation to Burgers equation with $N=160$ and $\epsilon=1 / 160^{2}$ : a) solution of the equation with triangulation of the domain (represented in red), b) iso-values of the solution.

In the first experiment we have chosen two scenarios. In the first one $\epsilon=$ $1 / N$ and in the second one $\epsilon=1 / N^{2}$ with $u_{D}=-2 x(x-1)$ in both. Results are presented in Figure 2. Furthermore, to prove the convergence of the (3.1) we performed two convergence test (see Table 1), where referent solution $u_{R}$ has been computed on $N \times N=640^{2}$ grid.

In the second and third experiment we have chosen $u_{D}=H(x-0.5)$ and $u_{D}=H(0.5-x)$, respectively, where $H$ is the Heaviside function. Results are presented on Figure 3 where we observe propagation of the rarefaction wave, while on Figure 4 we have propagation of the shock wave. 
Table 1. Results of convergence test.

\begin{tabular}{llll}
\hline$N=1 / \epsilon$ & $\left\|u_{N}-u_{R}\right\|_{2} /\left\|u_{R}\right\|_{2}$ & $N=1 / \sqrt{\epsilon}$ & $\left\|u_{N}-u_{R}\right\|_{2} /\left\|u_{R}\right\|_{2}$ \\
\hline 10 & 0.179448 & 10 & 0.0539613 \\
20 & 0.130928 & 20 & 0.0137841 \\
40 & 0.076787 & 40 & 0.0038117 \\
80 & 0.038821 & 80 & 0.0010069 \\
160 & 0.0167232 & 160 & 0.00029879 \\
320 & 0.0054824 & 320 & 0.000093223 \\
\hline
\end{tabular}
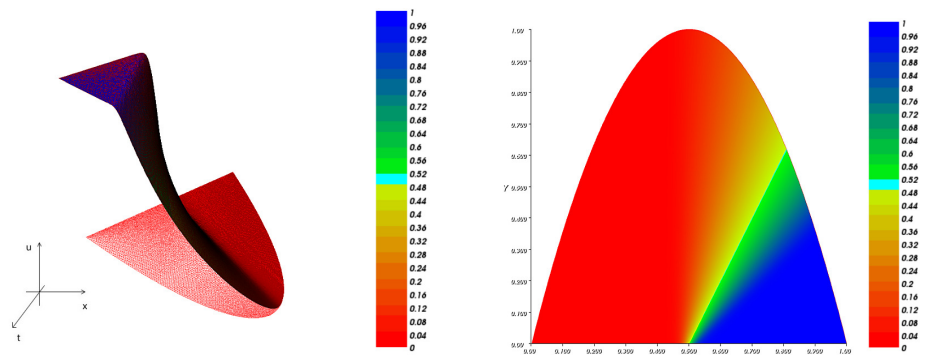

Figure 3. Numerical solution of elliptic approximation to Burgers equation demonstrating evolution of the rarefaction wave. Characteristics are plotted on the right plot.
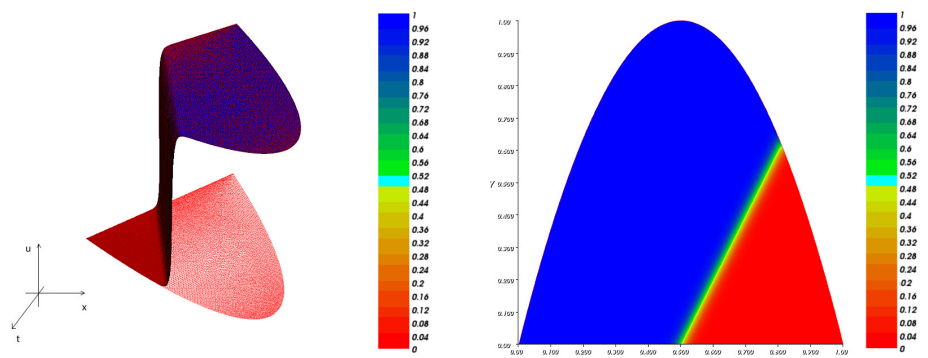

Figure 4. Numerical solution of elliptic approximation to Burgers equation demonstrating evolution of the shock wave. Characteristics are plotted on the right plot.

\section{Acknowledgements}

We would like to thank the referees for their generous help.

The research is supported by the bilateral project Multiscale Methods and Calculus of Variations between Croatia and Montenegro; by the Ministry of Science of Montenegro, project number 01-471; by the University of Zagreb through grant PP04/2015; by the Croatian Science Foundation, project number 9780 Weak convergence methods and applications (WeConMApp); and by the FP7 project Micro-local defect functional and applications (MiLDeFA) in the frame of the program Marie Curie FP7-PEOPLE-2011-COFUND. 


\section{References}

[1] J. Aleksić, D. Mitrovic and S. Pilipović. Hyperbolic conservation laws with vanishing nonlinear diffusion and linear dispersion in heterogeneous media. Journal of Evolution Equations, 9(4):809-828, 2009. http://dx.doi.org/10.1007/s00028009-0035-5.

[2] K. Ammar, P. Wittbold and J. Carrillo. Scalar conservation laws with general boundary condition and continuous flux function. Journal of Differential Equations, 228(1):111-139, 2006. http://dx.doi.org/10.1016/j.jde.2006.05.002.

[3] B. Andreianov and D. Mitrović. Entropy conditions for scalar conservation laws with discontinuous flux revisited. Annales de l'Institut Henri Poincare (C) Non Linear Analysis, 32(6):1307-1335, 2015. http://dx.doi.org/10.1016/j.anihpc.2014.08.002.

[4] B. Andreianov and K. Sbihi. Well-posedness of general boundary-value problems for scalar conservation laws. Transactions of the American Mathematical Society, 367:3763-3806, 2015. http://dx.doi.org/10.1090/S0002-9947-2015-059881\#sthash.4STac3SY.dpuf.

[5] C. Bardos, A.Y. Leroux and J.C. Nedelec. First order quasilinear equations with boundary conditions. Communications in Partial Differential Equations, 4(9):1017-1034, 1979. http://dx.doi.org/10.1080/03605307908820117.

[6] R. Borsche, R.M. Colombo and M. Garavello. Mixed systems: ODEsbalance laws. Journal of Differential Equations, 252(3):2311-2338, 2012. http://dx.doi.org/10.1016/j.jde.2011.08.051.

[7] R. Bürger, H. Frid and K.H. Karlsen. On the well-posedness of entropy solutions to conservation laws with a zero-flux boundary condition. Journal of Mathematical Analysis and Applications, 326(1):108-120, 2007. http://dx.doi.org/10.1016/j.jmaa.2006.02.072.

[8] R. Bürger and W. L. Wendland. Entropy boundary and jump conditions in the theory of sedimentation with compression. Mathematical Methods in the Applied Sciences, 21(9):865-882, 1998.

[9] G. M. Coclite and M. Garavello. Vanishing viscosity for mixed systems with moving boundaries. Journal of Functional Analysis, 264(7):1664-1710, 2013. http://dx.doi.org/10.1016/j.jfa.2013.01.010.

[10] G.M. Coclite, M. Garavello and B. Piccoli. Traffic flow on a road network. SIAM Journal on Mathematical Analysis, 36(6):1862-1886, 2005. http://dx.doi.org/10.1137/S0036141004402683.

[11] G.M. Coclite, K.H. Karlsen and Y.-S. Kwon. Initial-boundary value problems for conservation laws with source terms and the DegasperisProcesi equation. Journal of Functional Analysis, 257(12):3823-3857, 2009. http://dx.doi.org/10.1016/j.jfa.2009.09.022.

[12] R.J. DiPerna. Convergence of the viscosity method for isentropic gas dynamics. Communications in Mathematical Physics, 91(1):1-30, 1983. http://dx.doi.org/10.1007/BF01206047.

[13] L.C. Evans. Weak Convergence Methods for Nonlinear Partial Differential Equations. American Mathematical Society, 1990. CBMS Regional Conference Series in Mathematics. Volume 74

[14] H. Greenberg. An analysis of traffic flow. Operations Research, 7(1):79-85, 1959. http://dx.doi.org/10.1287/opre.7.1.79. 
[15] C. Imbert and J. Vovelle. A kinetic formulation for multidimensional scalar conservation laws with boundary conditions and applications. SIAM Journal on Mathematical Analysis, 36(1):214-232, 2004. http://dx.doi.org/10.1137/S003614100342468X.

[16] C.I. Kondo and P.G. LeFloch. Zero diffusion-dispersion limits for scalar conservation laws. SIAM Journal on Mathematical Analysis, 33(6):1320-1329, 2002. http://dx.doi.org/10.1137/S0036141000374269.

[17] Y.-S. Kwon and A. Vasseur. Strong traces for solutions to scalar conservation laws with general flux. Archive for Rational Mechanics and Analysis, 185(3):495513, 2007. http://dx.doi.org/10.1007/s00205-007-0055-7.

[18] M.J. Lighthill and G.B. Whitham. On kinematic waves. ii. a theory of traffic flow on long crowded roads. Proceedings of the Royal Society of London A: Mathematical, Physical and Engineering Sciences, 229(1178):317-345, 1955. http://dx.doi.org/10.1098/rspa.1955.0089.

[19] Y. Lu. Hyperbolic Conservation Laws and the Compensated Compactness Method. Chapman and Hall/CRC, 2002.

[20] E. Panov. On weak completeness of the set of entropy solutions to a scalar conservation law. SIAM Journal on Mathematical Analysis, 41(1):26-36, 2009. http://dx.doi.org/10.1137/080724587.

[21] E.Yu. Panov. On the Dirichlet problem for first order quasilinear equations on a manifold. Transactions of the American Mathematical Society, 363:2393-2446, 2011. http://dx.doi.org/10.1090/S0002-9947-2010-05016-0.

[22] H. J. Payne. Models of freeway traffic and control. In Frank C. Rieman(Ed.), Math. Models of Public Systems, Simulations Council Proceedings, pp. 51-60. Simulation Councils, Incorporated, 1971.

[23] P.I. Richards. Shock waves on the highway. Operations Research, 4(1):42-51, 1956. http://dx.doi.org/10.1287/opre.4.1.42.

[24] M.E. Schonbek. Convergence of solutions to nonlinear dispersive equations. Communications in Partial Differential Equations, 7(8):959-1000, 1982. http://dx.doi.org/10.1080/03605308208820242.

[25] I.S. Strub and A.M. Bayen. Mixed initial-boundary value problems for scalar conservation laws: Application to the modeling of transportation networks. In J.P. Hespanha and A. Tiwari(Eds.), Hybrid Systems: Computation and Control, volume 3927 of Lecture Notes in Computer Science, pp. 552-567, Berlin, Heidelberg, 2006. Springer Berlin Heidelberg. http://dx.doi.org/10.1007/11730637_41.

[26] M. Svärd and S. Mishra. Entropy stable schemes for initial-boundary-value conservation laws. Zeitschrift für angewandte Mathematik und Physik, 63(6):9851003, 2012. http://dx.doi.org/10.1007/s00033-012-0216-x.

[27] L. Tartar. Compensated compactness and applications to partial differential equations. In Nonlinear Analysis and Mechanics: Heriot-Watt Symposium, Vol. IV, Research Notes in Mathematics 39, pp. 136-212. Pitman, Boston, MassLondon, 1979. 